\title{
A special perturbation method in orbital dynamics
}

\author{
Jesús Peláez • José Manuel Hedo • \\ Pedro Rodríguez de Andrés
}

Received: 13 April 2006 / Revised: 31 July 2006 /

Accepted: 13 October 2006

(C) Springer Science+Business Media B.V. 2006

\begin{abstract}
The special perturbation method considered in this paper combines simplicity of computer implementation, speed and precision, and can propagate the orbit of any material particle. The paper describes the evolution of some orbital elements based in Euler parameters, which are constants in the unperturbed problem, but which evolve in the time scale imposed by the perturbation. The variation of parameters technique is used to develop expressions for the derivatives of seven elements for the general case, which includes any type of perturbation. These basic differential equations are slightly modified by introducing one additional equation for the time, reaching a total order of eight. The method was developed in the Grupo de Dinámica de Tethers (GDT) of the UPM, as a tool for dynamic simulations of tethers. However, it can be used in any other field and with any kind of orbit and perturbation. It is free of singularities related to small inclination and/or eccentricity. The use of Euler parameters makes it robust. The perturbation forces are handled in a very simple way: the method requires their components in the orbital frame or in an inertial frame. A comparison with other schemes is performed in the paper to show the good performance of the method.
\end{abstract}

Keywords Special perturbations - Orbital dynamics · Numerical methods · Propagation of orbits

J. Peláez $(\varangle)$ · J. M. Hedo · P. Rodríguez de Andrés

ETSI Aeronáuticos, Technical University of Madrid (UPM),

$\mathrm{Pz}$ Cardenal Cisneros 3,

28040 Madrid, Spain

e-mail: j.pelaez@upm.es

J. M. Hedo

e-mail: josemanuel.hedo@upm.es 


\section{Introduction}

A special perturbation method determines the trajectory of celestial bodies by numerically integrating the equations of motion. In order to solve a given special perturbation problem several points should be clarified in advance: (i) the type of orbit, (ii) the operational requirements, (iii) the formulation of equations of motion, (iv) the numerical integration procedure, and (v) the available computing facilities (Roy 1988).

The method carried out in this paper involves a new formulation of equations of motion. This formulation is appropriate for any kind of orbit (almost circular, highly eccentric or parabolic-hyperbolic), and it can be used with different numerical integration procedures (single-step or multi-step, constant-step or step-changing).

At present, the determination of satellite orbits involves a steadily increasing precision. Centimeter accuracy over weeks are becoming usual in several applications of satellite constellations. Any special perturbation method facing these type of problems should exhibit high efficiency and computing speed.

The method shown in this paper was born in the field of dynamical simulation of tethers, where bead models are widely used. The cable is discretized using beads distributed along its length; neighbor beads are linked through forces modeling the elasticity and damping of the tether. The time evolution of the beads is obtained numerically, considering the perturbation forces acting on the tether. Sometimes, the simulation extends over several years. The complex interactions between the cable and its spatial environment require to optimize the propagators that constitute the core of the process. Bead models keep direct ties to physical effects involved in the simulation and they are preferable when the dynamics is not well known. They can be easily programmed and - for small number of beads - they are fast. The precision can be readily improved by increasing the number of beads.

The method is not exclusive to the tether field; quite the opposite, it is completely general and it can be used, practically, in any numerical problem of Celestial Mechanics or Orbital Dynamics. Nevertheless, due to its origin, it does not give preferential treatment to conservative perturbation forces which are the most usual in Celestial Mechanics (aerodynamic drag or electrodynamic forces acting on electrodynamic tethers are not conservative). This peculiarity does not hinder its use; in fact, it can be readily used since the perturbation forces are managed through their components in an inertial frame, which is practically the case of the most typical routines in Astrodynamics. It is our intention to further study this subject in the future, trying to obtain a specific formulation for the particular case of hamiltonian systems. For this type of systems there are modern numerical integration procedures which are compatible with the local symplectic geometry of their associated phase space. In our opinion, our method used together some of these integrators would give rise to an excellent numerical tool.

\section{Equations for the particle}

Let $E x_{1} y_{1} z_{1}$ be the inertial geocentric frame. Its origin is at the mass center of the Earth, its $E x_{1}$ axis toward the first Aries Point $(\Upsilon)$. The problem of determining the time evolution of the position vector $\mathbf{x}=\mathbf{x}(t)$ of a particle $O$ with mass $m$ in this 
reference is a 6-order differential problem. The equations governing its motion are:

$$
m \ddot{\mathbf{x}}=-\frac{m \mu}{|\mathbf{x}|^{3}} \mathbf{x}+\mathbf{F}_{\mathrm{p}}
$$

where $\mathbf{x}=\overrightarrow{\mathbf{E O}}$ is the position vector of the particle and $\mathbf{F}_{\mathrm{p}}$ is the perturbation force, which is defined as the vector resultant of all the forces except the main term of the gravitacional potential. The natural variables for the numerical integration of the system (1) are $x_{1}, y_{1}, z_{1}, \dot{x}_{1}, \dot{y}_{1}$ and $\dot{z}_{1}$, that is, the cartesian coordinates of $O$ and its time derivatives, which define its dynamical state. The solution of this system of equations has the form

$$
\mathbf{x}=\mathbf{x}\left(t ; \mathbf{x}_{0}, \dot{\mathbf{x}}_{0}\right)
$$

where, without loss of generality, position $\left(\mathbf{x}_{0}\right)$ and velocity $\left(\dot{\mathbf{x}}_{0}\right)$ of $O$ at $t=0$ (initial time) are taken as integration constants.

Let $\mathcal{R}=\{O ; \mathbf{i}, \mathbf{j}, \mathbf{k}\}$ be an orbital frame associated with the particle motion (see Fig. 1). Its origin $O$ is at the particle and its unit vectors $(\mathbf{i}, \mathbf{j}, \mathbf{k})$ are defined as follows

- $\mathbf{i}$, in the same direction and way of the position vector $\mathbf{x}$

- $\mathbf{k}$, contained in the osculating orbital plane defined by $E, \mathbf{x}$ and the velocity $\mathbf{v}=\dot{\mathbf{x}}$ of the particle, (in such a way that $\mathbf{k} \cdot \mathbf{v} \geq 0$ )

$-\mathbf{j}=\mathbf{k} \times \mathbf{i}$, to fulfill the right-handed condition.

Thus, if the particle evolution is known the orbital reference evolution is known as well, and it follows from the relations

$$
\mathbf{i}=\frac{\mathbf{x}}{|\mathbf{x}|}=\frac{\mathbf{x}}{R}, \quad \mathbf{j}=\frac{\mathbf{v} \times \mathbf{x}}{|\mathbf{v} \times \mathbf{x}|}=-\frac{\mathbf{h}}{h}, \quad \mathbf{k}=\mathbf{i} \times \mathbf{j}
$$

where $R(t)$ is the orbital distance $R=|\mathbf{x}|$ and $\mathbf{h}=\mathbf{x} \times \mathbf{v}$ is the specific angular momentum.

Let us consider a rigid body $S$ in free motion relative to the frame $E x_{1} y_{1} z_{1}$. Its time evolution is given by a 12-order problem: six variables associated with the dynamic state of one of its points and six additional variables for its attitude. However, the time

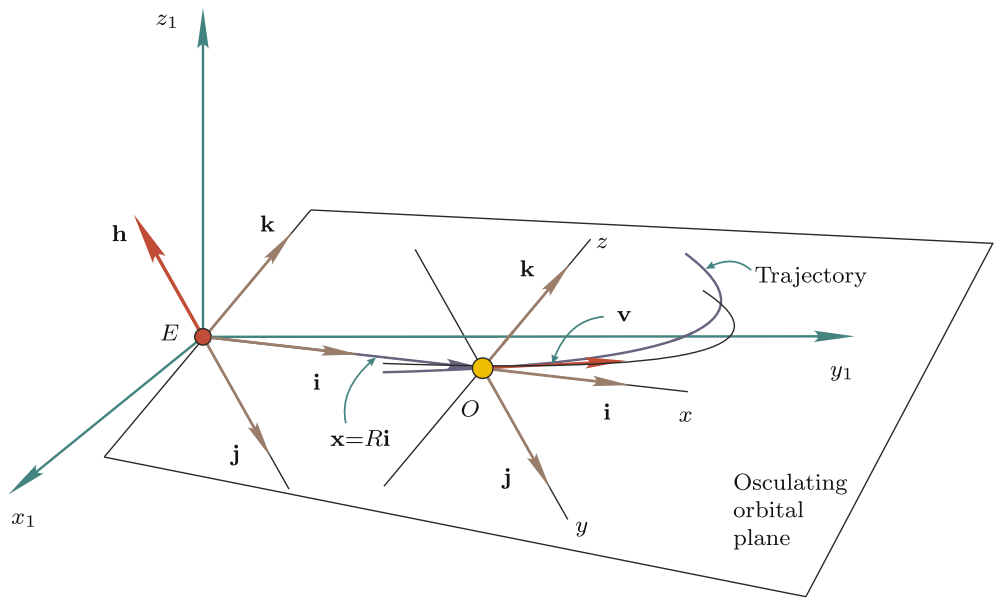

Fig. 1 Orbital reference 
evolution of the above orbital frame $\mathcal{R}$ is governed by a 6-order system, equivalent to the system (1) that governs the time evolution of the particle $O$. This is the basic starting point of the special perturbation method proposed in this paper.

Let $\boldsymbol{\omega}=p \mathbf{i}+q \mathbf{j}+r \mathbf{k}$ be the angular velocity of the orbital frame $\mathcal{R}$, and $\left(\psi_{1}, \theta_{1}, \varphi_{1}\right)$ three Euler angles providing its attitude relative to the frame $E x_{1} y_{1} z_{1}$. The time evolution of $\mathcal{R}$ is given by the time evolution of $R,(p, q, r)$ and $\left(\psi_{1}, \theta_{1}, \varphi_{1}\right)$. Seemingly, this is a 7 th order problem but actually is of 6 th order since, for the frame $\mathcal{R}$, the third component $(r)$ of the angular velocity $\omega$ is always zero. This fundamental property is deduced from the time derivatives of $(\mathbf{i}, \mathbf{j})$ unit vectors, that can be expressed in two ways: (i) from time derivative of Eq. 2 and, (ii) from elemental properties of the angular velocity of a rigid body.

$$
\begin{aligned}
& \frac{\mathrm{d} \mathbf{i}}{\mathrm{d} t}=\frac{\mathbf{v}}{R}-\frac{\dot{R}}{R^{2}} \mathbf{x}=\omega \times \mathbf{i}=r \mathbf{j}-q \mathbf{k} \\
& \frac{\mathrm{d} \mathbf{j}}{\mathrm{d} t}=\frac{\dot{h}}{h^{2}} \mathbf{h}-\frac{1}{h} \frac{\mathrm{d} \mathbf{h}}{\mathrm{d} t}=\omega \times \mathbf{j}=-r \mathbf{i}+p \mathbf{k} .
\end{aligned}
$$

The components of the angular velocity of $\mathcal{R}$ are deduced by identifying the corresponding values in both expressions

$$
p=-\frac{1}{h}\left(\mathbf{k} \cdot \frac{\mathrm{d} \mathbf{h}}{\mathrm{d} t}\right), \quad q=-\frac{1}{R}(\mathbf{v} \cdot \mathbf{k}), \quad r=\frac{1}{R}(\mathbf{v} \cdot \mathbf{j})=0
$$

With the help of the angular momentum equation

$$
\frac{\mathrm{dh}}{\mathrm{d} t}=\frac{1}{m}\left(\mathbf{x} \times \mathbf{F}_{\mathrm{p}}\right)
$$

which is readily deduced from Eq. (1), the components of $\omega$ take the form

$$
p=-\frac{R}{m h}\left(\mathbf{F}_{\mathrm{p}} \cdot \mathbf{j}\right), \quad q=-\frac{h}{R^{2}}, \quad r=0
$$

Thus, the third component $(r)$ is always null independently of the perturbation force acting on the particle.

Note that if the perturbation vanishes, $\left(\mathbf{F}_{\mathrm{p}}=\mathbf{0}\right)$, the motion of the particle is Keplerian: $\mathbf{h}$ is a constant vector and the law of areas $R^{2} \dot{\theta}=h$ is satisfied. In such a case, the components of the angular velocity are:

$$
p=0, \quad q=-\dot{\theta}, \quad r=0
$$

where $\theta$ is the true anomaly.

In order to obtain the time-evolution equations for the orbital frame, the time derivatives of the position vector $\mathbf{x}=R \mathbf{i}$ will be calculated

$$
\begin{aligned}
\frac{\mathrm{d} \mathbf{x}}{\mathrm{d} t} & =\dot{R} \mathbf{i}+R \boldsymbol{\omega} \times \mathbf{i} \\
\frac{\mathrm{d}^{2} \mathbf{x}}{\mathrm{d} t^{2}} & =\ddot{R} \mathbf{i}+R[\dot{\omega} \times \mathbf{i}+\omega \times(\omega \times \mathbf{i})]+2 \dot{R} \omega \times \mathbf{i}
\end{aligned}
$$

Equation 1 takes the form

$$
\ddot{R} \mathbf{i}+2 \dot{R} \omega \times \mathbf{i}+R\{\dot{\boldsymbol{\omega}} \times \mathbf{i}+\omega \times(\omega \times \mathbf{i})\}=-\frac{\mu}{R^{2}} \mathbf{i}+\frac{\mathbf{F}_{\mathrm{p}}}{m}
$$


and projecting on $\mathcal{R}$, we obtain the following equations

$$
\begin{aligned}
\ddot{R}-R q^{2}+\frac{\mu}{R^{2}} & =+\frac{1}{m}\left(\mathbf{F}_{\mathrm{p}} \cdot \mathbf{i}\right) \\
R p q & =+\frac{1}{m}\left(\mathbf{F}_{\mathrm{p}} \cdot \mathbf{j}\right) \\
\frac{\mathrm{d}}{\mathrm{d} t}\left(R^{2} q\right) & =-\frac{R}{m}\left(\mathbf{F}_{\mathrm{p}} \cdot \mathbf{k}\right)
\end{aligned}
$$

which provide $R(t), p(t), q(t)$ after the appropriate integration. From them, the evolution of $(\mathbf{i}, \mathbf{j}, \mathbf{k})$ can be obtained through the relations

$$
\begin{aligned}
& \frac{\mathrm{d} \mathbf{i}}{\mathrm{d} t}=\omega \times \mathbf{i}=-q \mathbf{k} \\
& \frac{\mathrm{d} \mathbf{j}}{\mathrm{d} t}=\omega \times \mathbf{j}=+p \mathbf{k} \\
& \frac{\mathrm{d} \mathbf{k}}{\mathrm{d} t}=\omega \times \mathbf{k}=+q \mathbf{i}-p \mathbf{j}
\end{aligned}
$$

These results are well known and they have been obtained in several places. The deduction given in (Deprit et al. 1994) is specially elegant.

\subsection{State variables}

The next step is to introduce non-dimensional variables and a more suitable set of generalized coordinates. Then, Euler parameters will be introduced to simplify the Eqs. 6-8.

Three characteristic magnitudes are introduced for length, time and mass; the remaining magnitudes are derived from them. The characteristic values used to obtain non-dimensional variables are summarized in Table 1 , where $R_{0}$ is the initial value of $R$ and $\omega_{0}$ is the angular frequency of the circular orbit at distance $R_{0}$ from $E$. We use the non-dimensional variables $\tau, r, P$ and $Q$ defined by

$$
\tau=\omega_{0} t, \quad R=R_{0} r, \quad q=\omega_{0} Q, \quad p=\omega_{0} P
$$

Note that, from now on, $r$ represents a non-dimensional distance and not the third component of the angular velocity, since $P$ and $Q$ are the only non-dimensional components different from zero of $\omega$.

The Eqs. 3-5 will be rewritten using $(r, u, \psi)$ as state variables; $r$ has been defined in (9); $u$ and $\psi$ are defined by:

$$
u=\frac{\mathrm{d} r}{\mathrm{~d} \tau}, \quad \psi=-r^{2} Q
$$

Table 1 Characteristic values used to introduce non-dimensional variables

\begin{tabular}{llllll}
\hline Length & Time & Mass & Velocity & Acceleration & Force \\
\hline$R_{0}$ & $\omega_{0}^{-1}=\sqrt{R_{0}^{3} / \mu}$ & $m$ & $R_{0} \omega_{0}$ & $R_{0} \omega_{0}^{2}$ & $m R_{0} \omega_{0}^{2}$ \\
\hline
\end{tabular}


When expressed in these state variables, the equations take the form:

$$
\begin{aligned}
\frac{\mathrm{d} r}{\mathrm{~d} \tau} & =u \\
\frac{\mathrm{d} u}{\mathrm{~d} \tau} & =\frac{\psi^{2}}{r^{3}}-\frac{1}{r^{2}}+\left(\mathbf{f}_{\mathrm{p}} \cdot \mathbf{i}\right) \\
\frac{\mathrm{d} \psi}{\mathrm{d} \tau} & =r\left(\mathbf{f}_{\mathrm{p}} \cdot \mathbf{k}\right)
\end{aligned}
$$

where

$$
\mathbf{f}_{\mathrm{p}}=\frac{1}{m R_{0} \omega_{0}^{2}} \mathbf{F}_{\mathrm{p}}
$$

is the non-dimensional value of the perturbation force. The non-dimensional components $(P, Q)$ of the angular velocity are given, in terms of the state variables, by the additional relations:

$$
\begin{aligned}
P & =-\frac{r}{\psi}\left(\mathbf{f}_{\mathrm{p}} \cdot \mathbf{j}\right) \\
Q & =-\frac{\psi}{r^{2}}
\end{aligned}
$$

Equation 12 shows that $\psi$ is constant in the unperturbed problem $\left(\mathbf{F}_{\mathrm{p}}=\mathbf{0}\right)$; its value coincides with the constant in the law of areas in non-dimensional form (see Eq. 14), that is, is the non-dimensional value of the modulus of the angular momentum vector.

\subsection{Euler parameters}

Two fundamental frames appear in the problem: (1) the inertial frame $E x_{1} y_{1} z_{1}$, whose unit vectors are denoted by $\left(\mathbf{i}_{1}, \mathbf{j}_{1}, \mathbf{k}_{1}\right)$, and (2) the orbital frame $\mathcal{R}$, whose unit vectors are denoted by $(\mathbf{i}, \mathbf{j}, \mathbf{k})$. The relation between the two sets of unit vectors is given by the equation ${ }^{1}$

$$
[\mathbf{i} \mathbf{j} \mathbf{k}]=\left[\mathbf{i}_{1} \mathbf{j}_{1} \mathbf{k}_{1}\right] \mathcal{Q}(t)
$$

Here $\mathcal{Q}$ is an special orthogonal matrix of third order which changes with time if the particle is moving. Only three of the nine elements of the matrix are independent. Usually the elements of $\mathcal{Q}$ are expressed in terms of three independent coordinates (e.g., Euler angles). From the viewpoint of numerical integration this election has a disadvantage: there is always a singular orientation where the matrix $\mathcal{Q}$ becomes singular. If the numerical integration approaches the singularity the simulation is jeopardized.

The minimum number of parameters needed to remove the singularity in the representation of $\mathcal{Q}$ is four. This is the reason why Euler parameters $\left(\varepsilon=\left(\varepsilon_{1}, \varepsilon_{2}, \varepsilon_{3}\right), \eta\right)$ are used in this paper. The $\mathcal{Q}$ matrix in terms of these parameters takes the form:

$$
\mathcal{Q}=\left[\begin{array}{ccc}
1-2\left(\varepsilon_{2}^{2}+\varepsilon_{3}^{2}\right), & 2 \varepsilon_{1} \varepsilon_{2}-2 \eta \varepsilon_{3}, & 2 \varepsilon_{1} \varepsilon_{3}+2 \eta \varepsilon_{2} \\
2 \varepsilon_{1} \varepsilon_{2}+2 \eta \varepsilon_{3}, & 1-2\left(\varepsilon_{1}^{2}+\varepsilon_{3}^{2}\right), & 2 \varepsilon_{2} \varepsilon_{3}-2 \eta \varepsilon_{1} \\
2 \varepsilon_{1} \varepsilon_{3}-2 \eta \varepsilon_{2}, & 2 \varepsilon_{3} \varepsilon_{2}+2 \eta \varepsilon_{1}, & 1-2\left(\varepsilon_{1}^{2}+\varepsilon_{2}^{2}\right)
\end{array}\right]
$$

\footnotetext{
1 The notation uses some matrices, such as $\left[\mathbf{i}_{1} \mathbf{j}_{1} \mathbf{k}_{1}\right]$, which are no true matrices (matrix elements are scalars but not vectors). In algebraic operations these pseudo-matrices obey the same algebra rules, mutatis mutandi, than true matrices. This notation is easy to use and causes no error if everyone is forewarned. 
Following Euler's theorem, the transformation from the base $\left(\mathbf{i}_{1}, \mathbf{j}_{1}, \mathbf{k}_{1}\right)$ into the base $(\mathbf{i}, \mathbf{j}, \mathbf{k})$ by means of $\mathcal{Q}$ represents a rotation of value $\phi$ around a spatial direction defined by a unit vector a. Euler parameters define these geometric elements by relations:

$$
\varepsilon=\mathbf{a} \sin \frac{\phi}{2}, \quad \eta=\cos \frac{\phi}{2}
$$

If $p$ and $q$ are known, Eqs. $6-8$ provide the time evolution of $(\mathbf{i}, \mathbf{j}, \mathbf{k})$, unit vectors of the orbital frame $\mathcal{R}$. The time evolution of Euler parameters is given by the equations:

$$
\frac{\mathrm{d} \boldsymbol{\varepsilon}}{\mathrm{d} t}=\frac{1}{2}(\varepsilon \times \omega+\eta \omega), \quad \frac{\mathrm{d} \eta}{\mathrm{d} t}=-\frac{1}{2} \boldsymbol{\varepsilon} \cdot \boldsymbol{\omega}
$$

where all vectors have to be expressed in the orbital frame $(\mathbf{i}, \mathbf{j}, \mathbf{k})$. The Eqs. $6-8$ in terms of non-dimensional variables take the form:

$$
\begin{aligned}
\frac{\mathrm{d} \varepsilon_{1}}{\mathrm{~d} \tau} & =+\frac{1}{2} P \eta-\frac{1}{2} Q \varepsilon_{3} \\
\frac{\mathrm{d} \varepsilon_{3}}{\mathrm{~d} \tau} & =-\frac{1}{2} P \varepsilon_{2}+\frac{1}{2} Q \varepsilon_{1} \\
\frac{\mathrm{d} \varepsilon_{2}}{\mathrm{~d} \tau} & =+\frac{1}{2} P \varepsilon_{3}+\frac{1}{2} Q \eta \\
\frac{\mathrm{d} \eta}{\mathrm{d} \tau} & =-\frac{1}{2} P \varepsilon_{1}-\frac{1}{2} Q \varepsilon_{2}
\end{aligned}
$$

where non-dimensional components $(P, Q)$ of angular velocity $\omega$ are given by relations (13-14) and depend on the perturbation force $\mathbf{f}_{\mathrm{p}}$.

Since Euler parameters fulfil the condition

$$
\varepsilon_{1}^{2}+\varepsilon_{2}^{2}+\varepsilon_{3}^{2}+\eta^{2}=1
$$

only three of the four Eqs. 15-18 are independent. Nevertheless, relation 19 is not used to reduce the system order, but to check the quality of numerical solution obtained by integrating Eqs. 15-18. Following (19), the renormalization of the quaternion could be performed when needed.

\subsection{Scheme of method generation}

Equations $10-14$ and $15-18$ are the starting point of the process that leads to the integration method of this paper. The scheme followed can be summarized in three basic points:

- A fictitious time $\sigma$ is introduced with a change of independent variable, similar to the Sundmann transformation (Bond and Allman 1996). It is the first step in regularization of the two-body problem (Stiefel and Scheifele 1971). The change is defined by:

$$
\frac{\mathrm{d} \sigma}{\mathrm{d} \tau}=-Q(\sigma) \Rightarrow \tau=-\int_{\sigma_{0}}^{\sigma} \frac{\mathrm{d} \sigma}{Q(\sigma)}=\int_{\sigma_{0}}^{\sigma} \frac{r^{2} \mathrm{~d} \sigma}{\psi}
$$

- Then, the analytical solution of the unperturbed problem is obtained

$$
\mathbf{X}=\mathbf{X}(\sigma ; \mathbf{E})
$$


Here $\mathbf{X}$ represents the state of the system and $\mathbf{E}$ the six integration constants (orbital elements in broad sense) involved in the general solution of the unperturbed problem.

- The variation of constants method is used to solve the perturbed problem. Its solution is expressed in the form of (21), but considering orbital elements as unknown functions of $\sigma, \mathbf{E}=\mathbf{E}(\sigma)$ (they are not constant now). This is equivalent to taking (21) as a change of variables that expresses the state of the system $\mathbf{X}$ in terms of orbital elements $\mathbf{E}$. Such a change allows the equations of the perturbed problem to be rewritten in terms of $\mathbf{E}$ as follows:

$$
\frac{\mathrm{d} \mathbf{E}}{\mathrm{d} \sigma}=\mathbf{G}\left(\sigma, \mathbf{E}, \mathbf{f}_{\mathrm{p}}\right), \quad \mathbf{G}(\sigma, \mathbf{E}, \mathbf{0})=\mathbf{0}
$$

The right hand side of these equations cancels when the perturbation vanishes.

This basic scheme is developed below, first with Eqs. 10-14 and then with Eqs. 15-18.

\section{First set of equations}

The Eqs. 10-14 are rewritten taking $\sigma$, defined in (20), as independent variable. At the same time, instead of $r$ we take a new state variable

$$
z=\frac{1}{r}
$$

The next set of equations is obtained:

$$
\begin{array}{lll}
\frac{\mathrm{d} \tau}{\mathrm{d} \sigma}=\frac{r^{2}}{\psi} & \rightarrow & \frac{\mathrm{d} \tau}{\mathrm{d} \sigma}=\frac{1}{z^{2} \psi} \\
\frac{\mathrm{d} r}{\mathrm{~d} \sigma}=u \frac{r^{2}}{\psi} & \rightarrow & \frac{\mathrm{d} z}{\mathrm{~d} \sigma}=-\frac{u}{\psi} \\
\frac{\mathrm{d} u}{\mathrm{~d} \sigma}=\frac{\psi}{r}-\frac{1}{\psi}+\frac{r^{2}}{\psi}\left(\mathbf{f}_{\mathrm{p}} \cdot \mathbf{i}\right) & \rightarrow & \frac{\mathrm{d} u}{\mathrm{~d} \sigma}=z \psi-\frac{1}{\psi}+\frac{1}{z^{2} \psi}\left(\mathbf{f}_{\mathrm{p}} \cdot \mathbf{i}\right) \\
\frac{\mathrm{d} \psi}{\mathrm{d} \sigma}=\frac{r^{3}}{\psi}\left(\mathbf{f}_{\mathrm{p}} \cdot \mathbf{k}\right) & \rightarrow & \frac{\mathrm{d} \psi}{\mathrm{d} \sigma}=\frac{1}{z^{3} \psi}\left(\mathbf{f}_{\mathrm{p}} \cdot \mathbf{k}\right)
\end{array}
$$

The following relations, which give the components of angular velocity $\omega$ as functions of the state of the system, must be added

$$
\begin{aligned}
P & =-\frac{1}{z \psi}\left(\mathbf{f}_{\mathrm{p}} \cdot \mathbf{j}\right) \\
Q & =-\psi z^{2}
\end{aligned}
$$

\subsection{Unperturbed problem}

In the unperturbed problem the Eqs. 22 can be analytically integrated to yield:

$$
\begin{aligned}
z & =\frac{1}{\psi_{0}^{2}}+A \cos \sigma+B \sin \sigma \\
u & =\psi_{0}(A \sin \sigma-B \cos \sigma) \\
\psi & =\psi_{0}
\end{aligned}
$$


where $\psi_{0}, A$ and $B$ are integration constants depending on the initial conditions. This solution corresponds to the classical Keplerian motion.

Now, the independent variable $\sigma$ has a precise geometrical meaning: is the true anomaly of the Keplerian orbit followed by the particle. In fact, this result can be readily obtained by comparing the law of areas with the first of Eqs. 22

$$
R^{2} \frac{\mathrm{d} \theta}{\mathrm{d} t}=C \quad \leftrightarrow \quad r^{2} \frac{\mathrm{d} \sigma}{\mathrm{d} \tau}=\psi_{0}
$$

As consequence: $\sigma \equiv \theta$, and the initial condition for $\sigma$ emerges immediately from this: $\sigma(\tau=0)=\theta_{0}$; here $\theta_{0}$ is the initial true anomaly of the particle. This initial condition is the same in both problems, perturbed and unperturbed, since it does not depend on presence or absence of perturbation.

The introduction of the $z=1 / r$ variable is due to a well known fact. In the two-body problem, the second formula of Binet for central motions

$$
\gamma=-\frac{C^{2}}{r^{2}}\left\{\frac{\mathrm{d}^{2}}{\mathrm{~d} \theta^{2}}\left(\frac{1}{r}\right)+\frac{1}{r}\right\}
$$

provides a linear differential equation in $z$ which determines the trajectory of the particle. This linearization is a basic goal of all the processes used to regularize the equations of motion (Deprit et al. 1994).

The first of Eqs. 22 also provides, by means of an additional integration, the relation between the non-dimensional time $\tau$ and the true anomaly $\sigma$. Depending on the type of Keplerian orbit (elliptic, parabolic or hyperbolic) it could take three different forms. Nevertheless, such a relation is not interesting in this context.

\subsection{Perturbed problem}

Guided by the solution (25-27) of the unperturbed problem, a solution of the perturbed problem is sought in the form

$$
\begin{aligned}
z & =\frac{1}{\psi^{2}(\sigma)}+A(\sigma) \cos \sigma+B(\sigma) \sin \sigma \\
u & =\psi(\sigma)(A(\sigma) \sin \sigma-B(\sigma) \cos \sigma) \\
\psi & =\psi(\sigma)
\end{aligned}
$$

Here $\psi(\sigma), A(\sigma)$ and $B(\sigma)$ are unknown functions of $\sigma$ to be determined as part of the solution. It is equivalent to considering Eqs. 25-27 as a change of variable that permits to express the dynamical state of the system in terms of the generalized coordinates $\psi, A$ and $B$.

If these relations are introduced in Eqs. 22, the equations governing the evolution of $\psi(\sigma), A(\sigma)$ and $B(\sigma)$ are obtained. Thereby we get the system

$$
\begin{aligned}
& \frac{\mathrm{d} \psi}{\mathrm{d} \sigma}=\frac{1}{z^{3} \psi}\left(\mathbf{f}_{\mathrm{p}} \cdot \mathbf{k}\right) \\
& \frac{\mathrm{d} A}{\mathrm{~d} \sigma}=+\frac{\sin \sigma}{\psi^{2} z^{2}}\left(\mathbf{f}_{\mathrm{p}} \cdot \mathbf{i}\right)+\frac{1}{\psi} \frac{\mathrm{d} \psi}{\mathrm{d} \sigma}\left\{\cos \sigma\left(\frac{1}{\psi^{2}}+z\right)-A\right\} \\
& \frac{\mathrm{d} B}{\mathrm{~d} \sigma}=-\frac{\cos \sigma}{\psi^{2} z^{2}}\left(\mathbf{f}_{\mathrm{p}} \cdot \mathbf{i}\right)+\frac{1}{\psi} \frac{\mathrm{d} \psi}{\mathrm{d} \sigma}\left\{\sin \sigma\left(\frac{1}{\psi^{2}}+z\right)-B\right\}
\end{aligned}
$$




$$
\frac{\mathrm{d} \tau}{\mathrm{d} \sigma}=\frac{1}{z^{2} \psi}
$$

whose second members include the perturbation force $\mathbf{f}_{\mathrm{p}}$ that is a function of the state of the system. To calculate the right hand sides of these expressions, the following relations must be considered

$$
\begin{aligned}
z & =\frac{1}{\psi^{2}(\sigma)}+A(\sigma) \cos \sigma+B(\sigma) \sin \sigma \\
\frac{\mathrm{d} z}{\mathrm{~d} \sigma} & =-A(\sigma) \sin \sigma+B(\sigma) \cos \sigma
\end{aligned}
$$

which are satisfied at any time. Notice that, if $\mathbf{f}_{\mathrm{p}}$ vanishes in Eqs. 28-31, we recover the unperturbed problem for which $\psi, A$ and $B$ take constant values.

\section{Second set of equations}

Taking $\sigma$ as the independent variable, Eqs. $15-18$ become

$$
\begin{aligned}
\frac{\mathrm{d} \varepsilon_{1}}{\mathrm{~d} \sigma} & =-\frac{1}{2} \lambda(\sigma) \eta+\frac{1}{2} \varepsilon_{3} \\
\frac{\mathrm{d} \varepsilon_{3}}{\mathrm{~d} \sigma} & =+\frac{1}{2} \lambda(\sigma) \varepsilon_{2}-\frac{1}{2} \varepsilon_{1} \\
\frac{\mathrm{d} \varepsilon_{2}}{\mathrm{~d} \sigma} & =-\frac{1}{2} \lambda(\sigma) \varepsilon_{3}-\frac{1}{2} \eta \\
\frac{\mathrm{d} \eta}{\mathrm{d} \sigma} & =+\frac{1}{2} \lambda(\sigma) \varepsilon_{1}+\frac{1}{2} \varepsilon_{2}
\end{aligned}
$$

where the function $\lambda(\sigma)$, defined by the relation

$$
\lambda(\sigma)=\frac{P}{Q}=\frac{1}{\psi^{2} z^{3}} \cdot\left(\mathbf{f}_{\mathrm{p}} \cdot \mathbf{j}\right)
$$

is the ratio between the two components of angular velocity $\omega$ of the $\mathcal{R}$ frame. $\lambda(\sigma)$ can be singular if $z=0$ or $\psi=0$. The case $z=0$ appears when the trajectory reaches a point at the infinite $(r=\infty)$; the case $\psi=0$ appears when the angular momentum of the particle vanishes, that is, if the velocity is contained into the local vertical and the motion of the particle is rectilinear. None of these situations are presented in most of the problems of orbital dynamics. Nevertheless, the hyperbolic case would lead to a possible singularity when the particle approaches the asymptote, with $\lambda$ taking increasing positive values. However, this is a very strange situation because the out-ofplane component of the perturbation, $\left(\mathbf{f}_{\mathrm{p}} \cdot \mathbf{j}\right)$, would be different from zero. Note that when the particle moves away from the attraction center, usually the perturbations goes to zero quickly and $\lambda$ does not grow. The exception to this rule appears when the particle approaches another attraction center, which becomes more and more important; in such a case, the simulation requires to change from the original primary to the new primary at a certain moment. In any case, an asymptotic solution could be obtained in the limit $\lambda \rightarrow \infty$, which permits to overcome this difficulty, but for the sake of brevity it is not developed in these pages. 


\subsection{Unperturbed problem}

In the unperturbed problem $\lambda(\sigma) \equiv 0$, since $\mathbf{f}_{\mathrm{p}}=\mathbf{0}$. Eqs. $34-37$ take the form

$$
\begin{array}{ll}
\frac{\mathrm{d} \varepsilon_{1}}{\mathrm{~d} \sigma}=+\frac{1}{2} \varepsilon_{3}, & \frac{\mathrm{d} \varepsilon_{2}}{\mathrm{~d} \sigma}=-\frac{1}{2} \eta \\
\frac{\mathrm{d} \varepsilon_{3}}{\mathrm{~d} \sigma}=-\frac{1}{2} \varepsilon_{1}, & \frac{\mathrm{d} \eta}{\mathrm{d} \sigma}=+\frac{1}{2} \varepsilon_{2}
\end{array}
$$

and-changing the order of Euler parameters slightly - they can be analytically integrated to yield

$$
\left\{\begin{array}{c}
\varepsilon_{1} \\
\varepsilon_{3} \\
\varepsilon_{2} \\
\eta
\end{array}\right\}=\mathcal{M}\left(\sigma-\sigma_{0}\right)\left\{\begin{array}{c}
\varepsilon_{1}^{0} \\
\varepsilon_{3}^{0} \\
\varepsilon_{2}^{0} \\
\eta^{0}
\end{array}\right\}
$$

where $\mathcal{M}(\zeta)$ is an orthogonal 4-sized matrix, given by

$$
\mathcal{M}(\zeta)=\left[\begin{array}{cccc}
\cos (\zeta / 2) & \sin (\zeta / 2) & 0 & 0 \\
-\sin (\zeta / 2) & \cos (\zeta / 2) & 0 & 0 \\
0 & 0 & \cos (\zeta / 2) & -\sin (\zeta / 2) \\
0 & 0 & \sin (\zeta / 2) & \cos (\zeta / 2)
\end{array}\right]
$$

Note that $\mathcal{M}(0) \equiv I$, the 4-sized identity matrix. In (39) $\sigma_{0}$ represents the initial value of $\sigma$ and it coincides with the initial value $\theta_{0}$ of the true anomaly of the particle (in its initial osculating orbit). Values $\varepsilon_{1}^{0}, \varepsilon_{3}^{0}, \varepsilon_{2}^{0}$ and $\eta^{0}$ are integration constants that define the attitude of the orbital frame $\mathcal{R}$ at the initial time.

Note that the position of orbital frame $\mathcal{R}$ changes with time starting from an initial position. The solution (39) relates the attitude of $\mathcal{R}$ in a generic time with the attitude of the departure frame $\mathcal{R}_{0}$, namely, the frame $\mathcal{R}$ at the initial time $(t=0)$.

\subsection{Perturbed problem}

To approach the perturbed problem, it is appropriate to write Eqs. 34-37 in matricial form

$$
\left\{\begin{array}{l}
\mathrm{d} \varepsilon_{1} / \mathrm{d} \sigma \\
\mathrm{d} \varepsilon_{3} / \mathrm{d} \sigma \\
\mathrm{d} \varepsilon_{2} / \mathrm{d} \sigma \\
\mathrm{d} \eta / \mathrm{d} \sigma
\end{array}\right\}=S(\sigma)\left\{\begin{array}{c}
\varepsilon_{1} \\
\varepsilon_{3} \\
\varepsilon_{2} \\
\eta
\end{array}\right\}
$$

where matrix $S(\sigma)$ is

$$
S(\sigma)=\frac{1}{2}\left[\begin{array}{cccc}
0 & 1 & 0 & -\lambda(\sigma) \\
-1 & 0 & \lambda(\sigma) & 0 \\
0 & -\lambda(\sigma) & 0 & -1 \\
\lambda(\sigma) & 0 & 1 & 0
\end{array}\right]
$$


In the perturbed problem we seek a solution of Eq. 40 as follows

$$
\left\{\begin{array}{c}
\varepsilon_{1} \\
\varepsilon_{3} \\
\varepsilon_{2} \\
\eta
\end{array}\right\}=\mathcal{M}\left(\sigma-\sigma_{0}\right)\left\{\begin{array}{c}
\varepsilon_{1}^{0}(\sigma) \\
\varepsilon_{3}^{0}(\sigma) \\
\varepsilon_{2}^{0}(\sigma) \\
\eta^{0}(\sigma)
\end{array}\right\}
$$

where $\varepsilon_{1}^{0}(\sigma), \varepsilon_{3}^{0}(\sigma), \varepsilon_{2}^{0}(\sigma)$ and $\eta^{0}(\sigma)$ are functions of $\sigma$ to be determined as part of the solution. This is equivalent to considering Eq. 39 as a change of variables that permits to express the dynamical state of the system in terms of generalized coordinates $\varepsilon_{1}^{0}$, $\varepsilon_{3}^{0}, \varepsilon_{2}^{0}$ and $\eta^{0}$.

Introducing expressions (41) into Eq. 40, after some algebra, these take the form

$$
\left\{\begin{array}{l}
\mathrm{d} \varepsilon_{1}^{0}(\sigma) / \mathrm{d} \sigma \\
\mathrm{d} \varepsilon_{3}^{0}(\sigma) / \mathrm{d} \sigma \\
\mathrm{d} \varepsilon_{2}^{0}(\sigma) / \mathrm{d} \sigma \\
\mathrm{d} \eta^{0}(\sigma) / \mathrm{d} \sigma
\end{array}\right\}=F(\sigma)\left\{\begin{array}{c}
\varepsilon_{1}^{0}(\sigma) \\
\varepsilon_{3}^{0}(\sigma) \\
\varepsilon_{2}^{0}(\sigma) \\
\eta^{0}(\sigma)
\end{array}\right\}
$$

where the matrix

$$
F(\sigma)=\mathcal{M}^{T}\left(\sigma-\sigma_{0}\right)\left[S(\sigma) \mathcal{M}\left(\sigma-\sigma_{0}\right)-\frac{\mathrm{d} \mathcal{M}}{\mathrm{d} \sigma}\left(\sigma-\sigma_{0}\right)\right]
$$

can be written, in terms of the value $\Omega=\sigma-\sigma_{0}$, as

$$
F(\sigma)=\frac{\lambda(\sigma)}{2}\left[\begin{array}{cccc}
0 & 0 & -\sin \Omega & -\cos \Omega \\
0 & 0 & \cos \Omega & -\sin \Omega \\
\sin \Omega & -\cos \Omega & 0 & 0 \\
\cos \Omega & \sin \Omega & 0 & 0
\end{array}\right]
$$

After some development, the equations take the form

$$
\begin{aligned}
& \frac{\mathrm{d} \varepsilon_{1}^{0}}{\mathrm{~d} \sigma}=-\frac{\lambda(\sigma)}{2}\left\{\sin \left(\sigma-\sigma_{0}\right) \varepsilon_{2}^{0}+\cos \left(\sigma-\sigma_{0}\right) \eta^{0}\right\} \\
& \frac{\mathrm{d} \varepsilon_{2}^{0}}{\mathrm{~d} \sigma}=+\frac{\lambda(\sigma)}{2}\left\{\sin \left(\sigma-\sigma_{0}\right) \varepsilon_{1}^{0}-\cos \left(\sigma-\sigma_{0}\right) \varepsilon_{3}^{0}\right\} \\
& \frac{\mathrm{d} \varepsilon_{3}^{0}}{\mathrm{~d} \sigma}=+\frac{\lambda(\sigma)}{2}\left\{\cos \left(\sigma-\sigma_{0}\right) \varepsilon_{2}^{0}-\sin \left(\sigma-\sigma_{0}\right) \eta^{0}\right\} \\
& \frac{\mathrm{d} \eta^{0}}{\mathrm{~d} \sigma}=+\frac{\lambda(\sigma)}{2}\left\{\cos \left(\sigma-\sigma_{0}\right) \varepsilon_{1}^{0}+\sin \left(\sigma-\sigma_{0}\right) \varepsilon_{3}^{0}\right\}
\end{aligned}
$$

and should be integrated with suitable initial conditions.

Note again that if perturbations vanish then $\lambda \equiv 0$ and the generalized coordinates $\varepsilon_{1}^{0}, \varepsilon_{3}^{0}, \varepsilon_{2}^{0}$ and $\eta^{0}$ take constant values (the unperturbed problem results are recovered). The value of $\lambda$ only depends on the out-of-plane component of the perturbation (see (38)); when this component is small, the value of $\lambda$ will be small also, that is, $\lambda<<1$. In such a case, the evolution of $\varepsilon_{1}^{0}, \varepsilon_{3}^{0}, \varepsilon_{2}^{0}$ and $\eta^{0}$ take place in a large time scale.

Note that there are three frames in the problem: the inertial frame $E x_{1} y_{1} z_{1}$, the departure frame $\mathcal{R}_{0}$, whose attitude is defined by generalized coordinates $\varepsilon_{1}^{0}, \varepsilon_{3}^{0}, \varepsilon_{2}^{0}$ and $\eta^{0}$, and finally, the orbital frame $\mathcal{R}$ located at the actual position of the particle, whose attitude is defined by Euler parameters $\varepsilon_{1}, \varepsilon_{3}, \varepsilon_{2}$ and $\eta$. 
In the unperturbed problem, the departure frame $\mathcal{R}_{0}$ is fixed in frame $E x_{1} y_{1} z_{1}$ (it is also an inertial frame), but in the perturbed problem its position changes with time (slowly if the perturbation acting on the particle is small). In both cases the attitude of the orbital frame $\mathcal{R}$ (located at the actual position of the particle) is given by Eq. 41 . In the unperturbed problem $\mathcal{R}$ changes within an unique time scale (e.g., the orbital period in an elliptical orbit). In contrast, the time evolution of $\mathcal{R}$ involves two time scales in the perturbed problem. One of them defined by the osculating orbit (usually a fast time scale); the other one defined by the perturbation (usually a slow time scale). When the perturbation is small, the second is greater than the former. The relation between both orbital frames, $\mathcal{R}_{0}$ and $\mathcal{R}$, is clear: if the perturbation is removed at a given epoch, the particle will follow the osculating (Keplerian) orbit from this time forward. For this orbit, $\mathcal{R}_{0}$ is the departure frame at the epoch and $\mathcal{R}$ the orbital frame at a generic later time.

It is important to understand the structure of this propagator, since in order to calculate the perturbation (which determines the values of the right hand sides of equations), the orbital frame $\mathcal{R}$ located at the particle needs to be used. The integration process, however, only provides the orbital frame $\mathcal{R}_{0}$. The transfer from one frame to the other is carried out by Eq. 41.

All in all, the proposed method integrates Eqs. 28-31 and 42-45 starting from suitable initial conditions. Relations (32-33), (38), and (41) - satisfied at any time- must be considered in the integration process and also in the computation of the right hand sides of equations.

\subsection{A final change of variables}

A last change of variables leads to the final formulation:

$$
q_{1}=\psi A, \quad q_{2}=\psi B, \quad q_{3}=\frac{1}{\psi}
$$

The equations of this change are inspired by the structure of governing equations, where the derivatives of $\psi A$ and $\psi B$ appear in a natural form. Moreover, the total energy of the system in the unperturbed problem adopts the simplified form

$$
E=\frac{1}{2} m v^{2}-\frac{m \mu}{R}=\frac{m \mu}{2 R_{0}}\left\{q_{1}^{2}+q_{2}^{2}-q_{3}^{2}\right\}
$$

This change is not essential and it was initially guided by an erroneous argument. It is associated with a transformation -in the phase space of the unperturbed problem-from the original variables $R, p_{R}, \theta$ and $p_{\theta}\left(p_{R}\right.$ and $p_{\theta}$ are conjugate momenta) into the variables $q_{1}, q_{2}, q_{3}$ and $\theta$, given by equations

$$
\begin{aligned}
\frac{1}{R} & =q_{3}\left\{q_{3}+q_{1} \cos \theta+q_{2} \sin \theta\right\} \frac{1}{R_{0}} \\
\theta & =\theta \\
p_{R} & =m \sqrt{\frac{\mu}{R_{0}}}\left\{q_{1} \sin \theta-q_{2} \cos \theta\right\} \\
p_{\theta} & =m \sqrt{\mu R_{0}} \frac{1}{q_{3}}
\end{aligned}
$$

At the very beginning we were looking for a canonical transformation that, preserving the hamiltonian structure of equations, would improves the algorithm. Unfortunately, 
the above transformation is not canonical and does not bring any advantage in this sense. However, it leads to equations that exhibit slightly better performances than the original scheme; although the differences are not significant, it was finally adopted because there is no reason against using it.

\subsection{Summary of equations}

The method proposed is based on the following set of equations

$$
\begin{aligned}
\frac{\mathrm{d} \tau}{\mathrm{d} \sigma} & =+\frac{1}{q_{3} s^{2}} \\
\frac{\mathrm{d} q_{1}}{\mathrm{~d} \sigma} & =+\frac{\sin \sigma}{q_{3} s^{2}}\left(\mathbf{f}_{\mathrm{p}} \cdot \mathbf{i}\right)+\cos \sigma \frac{s+q_{3}}{q_{3} s^{3}}\left(\mathbf{f}_{\mathrm{p}} \cdot \mathbf{k}\right) \\
\frac{\mathrm{d} q_{2}}{\mathrm{~d} \sigma} & =-\frac{\cos \sigma}{q_{3} s^{2}}\left(\mathbf{f}_{\mathrm{p}} \cdot \mathbf{i}\right)+\sin \sigma \frac{s+q_{3}}{q_{3} s^{3}}\left(\mathbf{f}_{\mathrm{p}} \cdot \mathbf{k}\right) \\
\frac{\mathrm{d} q_{3}}{\mathrm{~d} \sigma} & =-\frac{1}{s^{3}}\left(\mathbf{f}_{\mathrm{p}} \cdot \mathbf{k}\right) \\
\frac{\mathrm{d} \varepsilon_{1}^{0}}{\mathrm{~d} \sigma} & =-\frac{\lambda(\sigma)}{2}\left\{\sin \left(\sigma-\sigma_{0}\right) \varepsilon_{2}^{0}+\cos \left(\sigma-\sigma_{0}\right) \eta^{0}\right\} \\
\frac{\mathrm{d} \varepsilon_{2}^{0}}{\mathrm{~d} \sigma} & =+\frac{\lambda(\sigma)}{2}\left\{\sin \left(\sigma-\sigma_{0}\right) \varepsilon_{1}^{0}-\cos \left(\sigma-\sigma_{0}\right) \varepsilon_{3}^{0}\right\} \\
\frac{\mathrm{d} \varepsilon_{3}^{0}}{\mathrm{~d} \sigma} & =+\frac{\lambda(\sigma)}{2}\left\{\cos \left(\sigma-\sigma_{0}\right) \varepsilon_{2}^{0}-\sin \left(\sigma-\sigma_{0}\right) \eta^{0}\right\} \\
\frac{\mathrm{d} \eta^{0}}{\mathrm{~d} \sigma} & =+\frac{\lambda(\sigma)}{2}\left\{\cos \left(\sigma-\sigma_{0}\right) \varepsilon_{1}^{0}+\sin \left(\sigma-\sigma_{0}\right) \varepsilon_{3}^{0}\right\}
\end{aligned}
$$

that should be integrated simultaneously with the relations needed to calculate the right hand sides of equations

$$
\begin{gathered}
\lambda(\sigma)=\frac{1}{q_{3} s^{3}}\left(\mathbf{f}_{\mathrm{p}} \cdot \mathbf{j}\right) \\
s=q_{3}+q_{1} \cos \sigma+q_{2} \sin \sigma \\
z=\frac{1}{r}=q_{3} \cdot s \\
\frac{\mathrm{d} r}{\mathrm{~d} \tau}=-\psi \frac{\mathrm{d} z}{\mathrm{~d} \sigma}=q_{1} \sin \sigma-q_{2} \cos \sigma \\
\left\{\begin{array}{c}
\varepsilon_{1} \\
\varepsilon_{3} \\
\varepsilon_{2} \\
\eta
\end{array}\right\}=\mathcal{M}\left(\sigma-\sigma_{0}\right)\left\{\begin{array}{c}
\varepsilon_{1}^{0}(\sigma) \\
\varepsilon_{3}^{0}(\sigma) \\
\varepsilon_{2}^{0}(\sigma) \\
\eta^{0}(\sigma)
\end{array}\right\} \\
1=\left(\varepsilon_{1}^{0}\right)^{2}+\left(\varepsilon_{2}^{0}\right)^{2}+\left(\varepsilon_{3}^{0}\right)^{2}+\left(\eta^{0}\right)^{2}
\end{gathered}
$$

\section{Advantages and drawbacks of the method}

The proposed method presents advantages of diverse nature which shares with other special perturbations methods. Some of them can be deduced directly from the 首 Springer 
theoretical formulation developed. Others can be deduced from different test carried out to check the goodness of the method. The main advantages and drawbacks found in the method are as follows

- Like the Cowell method, it has a unique formulation for the three types of orbits: elliptic, parabolic and hyperbolic. So, the singularity found in the proximity of parabolic motion when using different formulations for elliptic and hyperbolic orbits disappears.

- Unlike the Cowell's or Encke's methods, the error propagation is not exponential, (Bond and Allman 1996).

- It uses orbital elements as generalized coordinates, just as in the Lagrange's Planetary equations. As a consequence, the truncation error vanishes in the unperturbed problem and is scaled by the perturbation itself in the perturbed one.

- Unlike Lagrange's planetary equations, the method does not have singularities for small inclination and/or small eccentricities. The attitude of the orbital plane is determined by the Euler parameters which are free of singularities.

- The use of Euler parameters provides robustness and also permits easy autocorrection. When the sum of the second member of Eq. 60 differs from 1 more than a certain limit, Euler parameters can be normalized dividing by the module of the associated quaternion.

- When compared with the Cowell method, our procedure requires an additional operation, since it uses the components of perturbation forces in the orbital frame. However, this extra calculation, a dot product for each component, does not jeopardizes its performances as we shall show in the next section.

- When compared with more elaborated method (Kustaanheimo-Stiefel or Sperling-Burdet, for example) the procedure exhibits a easier programming. In any case, common perturbation models can be easily incorporated.

- A precise and fast simulator is obtained by using variable step routines with effective step control, as Runge-Kutta-Fehlberg or Dormand-Prince types. However, routines with fixed step also can de used without reduction in performances.

- It is not necessary to solve Kepler's equation in the elliptic case, nor the equivalent for hyperbolic and parabolic cases, since time is one of the dependent variables determined by the method itself.

- A drawback that can initially bewilder to the practitioner is the use of two different sets of Euler parameters: one of them associated with the actual position of the satellite, the other one related with the departure frame. The code is forced to change from one to other, with the help of Eqs. 55-59; however, this difficulty is not essential and it does not produces big problems.

Finally note that the method integrates a system of eight differential equations to solve a sixth-order problem and this is a characteristic shared with other regularization methods. Although increasing the order of the system in two unities can seem troublesome, there is no disadvantage, as can be shown by regularization methods and the results of the next section.

\section{Checking the method}

The method has been checked using the example $2 \mathrm{~b}$ of the book by Stiefel $\&$ Scheifele, pag. 122 of (Stiefel and Scheifele 1971); it deals with a satellite in an inclined $\left(i=30^{\circ}\right)$ 
elliptical orbit of great eccentricity $(e=0.95)$ and affected by only two perturbation forces: (1) the Earth oblateness and (2) the Lunar perturbation.

Parameters associated to Earth gravitation are

$$
J_{2}=1.08265 \times 10^{-3}, \quad R_{\mathrm{E}}=6371.22 \mathrm{~km}, \quad \mu=398601.0 \mathrm{~km}^{3} \mathrm{~s}^{-2}
$$

and Lunar perturbation is modeled with the force

$$
\mathbf{F}_{\mathrm{PL}}=-m \mu_{\mathrm{L}}\left\{\frac{\mathbf{R}-\boldsymbol{\rho}}{|\mathbf{R}-\rho|^{3}}+\frac{\rho}{\rho^{3}}\right\}
$$

where $\mathbf{R}$ and $\rho$ are the position vectors of satellite and Moon, respectively, in the inertial geocentric frame and $\mu_{\mathrm{L}}=4902.66 \mathrm{~km}^{3} \mathrm{~s}^{-2}$. The Moon position is given by the following ephemeris

$$
\rho=\rho\left\{\sin \Omega_{\mathrm{L}} t \mathbf{i}_{1}-\frac{\sqrt{3}}{2} \cos \Omega_{\mathrm{L}} t \mathbf{j}_{1}-\frac{1}{2} \cos \Omega_{\mathrm{L}} t \mathbf{k}_{1}\right\}
$$

which correspond to an inclined circular orbit, and where $\rho$ and $\Omega_{\mathrm{L}}$ take constants values

$$
\rho=384400 \mathrm{~km}, \quad \Omega_{\mathrm{L}}=2.665315780887 \times 10^{-6} \mathrm{~s}^{-1}
$$

The problem is determining the satellite's position after 50 revolutions (288.12768941 mean solar days), starting from the initial conditions

$$
\begin{aligned}
& \left(x_{1}, y_{1}, z_{1}\right)=(0.0,-5888.9727,-3400.0) \mathrm{km} \\
& \left(\dot{x}_{1}, \dot{y}_{1}, \dot{z}_{1}\right)=(10.691338,0.0,0.0) \mathrm{km} \mathrm{s}^{-1}
\end{aligned}
$$

which correspond to the perigee of the initial osculating orbit (at a distance $R=6,800 \mathrm{~km}$ from the Earth's center of mass). The most precise calculus of final position $\left(x_{1 f}, y_{1 f}, z_{1 f}\right)$ given in the reference (Stiefel and Scheifele 1971)

$$
\left(x_{1 f}, y_{1 f}, z_{1 f}\right)=(-24219.0503,227962.1064,129753.4424) \mathrm{km}
$$

was obtained by using a numerical integration scheme of 498 steps per revolution. The most precise final position achieved in our group is

$$
\begin{aligned}
& x_{1 f}=-24219.0501159 \mathrm{~km} \\
& y_{1 f}=227962.1063730 \mathrm{~km} \\
& z_{1 f}=129753.4424001 \mathrm{~km} .
\end{aligned}
$$

This problem is also used in reference (Bond and Allman 1996) in order to compare the performance of different methods. Ad hoc, the solution (61) given in (Stiefel and Scheifele 1971) is adopted as exact and the error is defined as the distance (in $\mathrm{km}$ ) between the final position provided by each method and the exact one. All the cases use variable step-size Runge-Kutta algorithms (we use here a Runge-KuttaFehlberg 4(5) adapted from the RKF 4(5) taken from the (Press et al. 1992)). The Table 2, taken from (Bond and Allman 1996), shows the results provided by different methods. We added the last column, which summarizes the results provided by the method presented in this paper.

Note that Cowell's method (Bond and Horn 1973) requires a greater number of steps per revolution (240) and, in spite of it, it is the less precise method, due to the exponential error propagation (Bond and Allman 1996). The KS's method (Bond 
Table 2 Comparison of special perturbation methods

\begin{tabular}{llllll}
\hline Method & $\begin{array}{l}\text { Stiefel \& } \\
\text { Scheifele }\end{array}$ & $\begin{array}{l}\text { Sperling \& } \\
\text { Burdet }\end{array}$ & $\begin{array}{l}\text { Kustaanheimo } \\
\text { \& Stiefel }\end{array}$ & Cowell & GDT \\
\hline$x(\mathrm{~km})$ & -24219.050 & -24218.818 & -24219.002 & -24182.152 & -24219.279 \\
$y(\mathrm{~km})$ & 227962.106 & 227961.915 & 227962.429 & 227943.989 & 227962.207 \\
$z(\mathrm{~km})$ & 129753.442 & 129753.343 & 129753.822 & 129744.270 & 129753.492 \\
Steps/rev & 500 & 62 & 62 & 240 & 62 \\
Error & & 0.318 & 0.501 & 42.5 & 0.250 \\
\hline
\end{tabular}

1974) is more accurate than the Cowell method but less than the Sperling-Burdet's method (Bond and Fraietta 1991). This last, considered as the most efficient in the book (Bond and Allman 1996), gives an error of $318 \mathrm{~m}$ using 62 steps per revolution. The method carried out in these pages clearly competes with Sperling-Burdet's method, since it provides a smaller error, of $250 \mathrm{~m}$, using 62 steps per revolution.

We would like to warn the reader about the accuracy of these calculations. It is obvious that any one of the methods considered in Table 2 is able to provide more accuracy if the integration is carried out using more steps per revolution. However, in a comparison like the one developed in this section the important parameter is the relative accuracy between the different methods for a given level of absolute accuracy.

A more detailed comparison with the Sperling-Burdet's method has been carried out. Both propagators have been coded and we have computed the former problem with them for different step error tolerances. In this comparison the exact solution is not the one given in the book (Stiefel and Scheifele 1971). Instead, we recalculated the solution two times using both propagators with the maximum accuracy; we took as exact solution the common part obtained in both calculations.

The computations have been done: (1) in the same computer (Intel Xeon $3056 \mathrm{MHz}$ microprocessor, 2 Gb RAM), (2) with the same compiler (Intel C++ 8.1.022), (3) with the same integrating algorithm (Runge-Kutta-Fehlberg (RKF) 7(8) of variable stepsize better than the RKF 4(5) taken from the (Press et al. 1992)), and (4) in the same computer conditions (processor load, etc). Moreover, to minimize the effect of uncontrolled factors on the computation time we have repeated the former task 30 times and we have obtained the mean value of runtime.

Figure 2 shows the results. The mean computation runtime is plotted in ordinates and the common logarithm of the norm of the error vector $(-\log (|\Delta \bar{x}|)$ in abscissas. This last quantity is a measure of the quality of the solution: it is approximately equivalent to the number of exact decimal digits of the solution plus one.

The plot shows better performances for the method carried out in these pages; it seems to be quicker for the same precision, or equivalently, it seems to be more accurate for identical computational time.

We believe these differences are mainly due to the lower order of our method. But there are other reasons also: in the Sperling-Burdet's method the calculation of the "second members" of equations requires to process perturbation forces through numerical treatments of some length; this also happens in similar methods based on regularization techniques as the KS's method. In our method however, forces hardly require manipulation. Note that the right hand sides of equations only include their components in the orbital frame $\mathcal{R}$, which are obtained by simple scalar products. Moreover, the simplicity of programming, joined to the clearness and the simplicity 


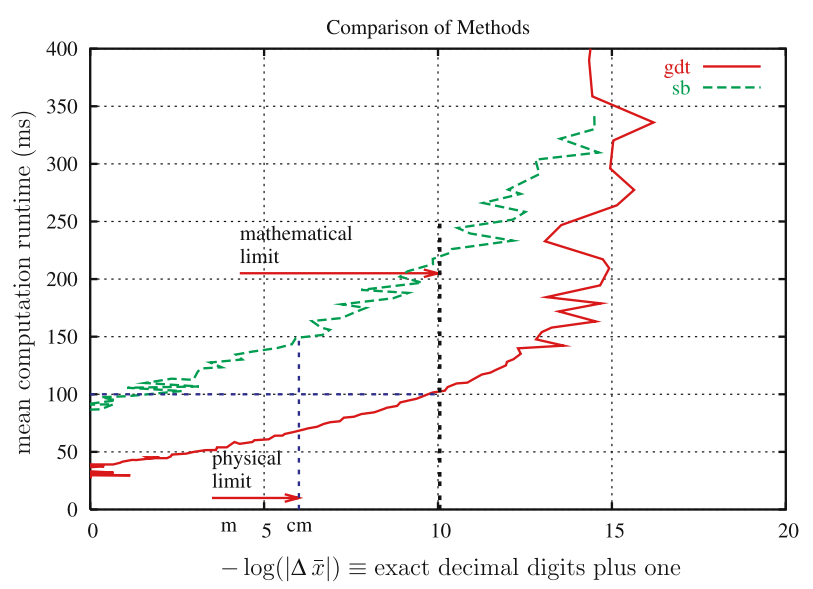

Fig. 2 Mean computation runtime versus quality parameter

of equations governing the evolution of Euler parameters, strengthens our conviction in the method's advantages.

A natural continuation of this work could be to validate this propagator. Precision Orbits Ephemerides (POE) are available for some satellites as LAGEOS, ETALON, GPS (Vallado 1997). The validation will require to determining the orbit of one of these test satellites using the same perturbation forces as in the POE and then comparing with the known solution. Such a detailed comparison is beyond the scope of this paper.

\section{Similar formulations}

An analysis of the available literature on the subject shows the existence of formulations similar to the one exposed in this article, although in different context. Particularly, almost identic formulations are used in the Department of Astronomy of the Cairo University, collected in references (Mohammed Adel Sharaf et al. 1992; Awad 1993, 1995). Likewise, similar schemes have been formulated in the Space Mechanics Group of the Zaragoza University (Spain), (Palacios and Calvo 1996).

In any case, our method has clear advantages in general when it is compared to other traditional methods. The reasons for this are no perfectly clear and are no simple to describe. The reader interested in deepening the subject can encounter an extensive analysis about regularization and linearization aspects in the reference (Deprit et al. 1994). These techniques had led the production of new perturbation methods in the last decades.

\section{Conclusions}

The formulation of a special perturbation method have been exposed in detail. The analysis of the structure of equations shows some of the intrinsic advantages of the method; they are 
- Unified formulation for the three kinds of orbits: elliptic, parabolic and hyperbolic

- Null truncation error in the unperturbed problem

- Truncation error scaled by perturbation in the perturbed problem

- It is not singular for small inclination nor small eccentricity

- There is no exponential error propagation

- Robustness and ease of programming.

It has been shown that the method exhibits indubitable advantages concerning precision, when it is compared to classical methods (the Cowell's or Encke's methods), as well as concerning computation runtime compared to more sophisticated methods (the KS or the Sperling-Burdet methods).

All these reasons make this perturbation method suitable to be used for general orbital motion, specially when a large number of particles should be propagated. This is the case, for example, of the tether simulators used in our group. They have to follow the time evolution of a great number of particles (bead models) and they are based in this method.

It should be notice that these advantages in runtime will be more pronounced in problems where the calculations of the perturbation forces does not take a significant percentage of the total runtime (per integration cycle). Obviously, when the most expensive task (numerically speaking) is the calculation of the perturbation forces, these differences become more and more damped.

Finally, along the paper we take the Earth as the primary attraction center. Obviously, this selection can be changed to any other attraction center (Sun, Jupiter, Moon, etc) without difficulty.

Acknowledgements This work is part of the research project Dynamics of Satellite Orbit Descent/Raise Using Electrodynamic Tethers (ESP2004-04376) supported by the Dirección General de Investigación (DGI) of the Spanish Ministry of Education and Science. Authors thank DGI by its support; they also thank Prof M. Ruiz for his help in preparing the final version of the manuscript.

\section{References}

Awad, M.E.-S.: Oblateneess and drag effects on the motion of satellites in the set of Eulerian redundant parameters. Earth, Moon Planets 62(2), 161-177 (1993)

Awad, M.E.-S.: Analytical solution to the perturbed $J_{2}$ motion of artificial satellites in terms of Euler parameters. Earth, Moon Planets 69(1), 1-12 (1995)

Bond, V.R.: The uniform, regular differential equations of the KS transformed perturbed two-body problem. Celest. Mech. 10(3), 303-318 (1974)

Bond, V.R., Allman, M.C.: Modern Astrodynamics: Fundamentals and Perturbation Methods. Princeton University Press, Princeton, New Jersey (1996)

Bond, V.R., Fraietta, M.F.: Elimination of secular terms from the differential equations for the elements of the perturbed two-body problem. In: Proceedings of the Flight Mechanics and Estimation Theory Symposium, NASA (1991)

Bond, V.R., Horn, M.K.: The Burdet formulation of the perturbed two body problem with total energy as an element. JSC Internal 73-FM-86, NASA (1973)

Deprit, A., Elipe, A., Ferrer, S.: Linearization: Laplace vs. Stiefel. Celest. Mech. Dyn. Astron. 58(2), 151-201 (1994)

Mohammed Adel Sharaf, Awad, M.E.-S., Najmuldeen, S.A.-S.A.: Motion of artificial satellites in the set of Eulerian redundant parameters (III). Earth, Moon Planets 56(2), 141-164 (1992)

Palacios, M., Calvo, C.: Ideal frames and regularization in numerical orbit computation. J. Astron. Sci. 44(1), 63-77 (1996) 
Press, W.H., Flannery, B.P., Teukolsky, S.A., Vetterling, W.T.: Numerical Recipes in C. The Art of Scientific Computing (2nd edn). Cambridge University Press, Cambridge, New York (1992)

Roy, A.E.: Orbital Motion. Adam Hilger (1988)

Stiefel, E.L., Scheifele, G.: Linear and Regular Celestial Mechanics. Springer Verlag, Berlin, Heidelberg, New York (1971)

Vallado, D.A.: Fundamentals of Astrodynamics and Applications. MacGraw-Hill (1997) 